\title{
Influence de la température sur le développement et la fécondité de quatre espèces de Cladocères planctoniques (Crustacés) en présence de ressources naturelles
}
A. Tifnouti1
R. Pourriot ${ }^{2}$
C. Rougier ${ }^{2}$

Mots clés : croissance, reproduction, Crustacés, Cladocères, température.

La croissance et la reproduction de quatre espèces de Cladocères (Daphnia pulex Leydig 1860, D. lumholtzi Sars 1886, Ceriodaphnia dubia Richard 1894 et Diaphanosoma brachyurum Liévin 1848) récoltés dans un lac réservoir marocain ont été étudiées à cinq températures $\left(10,15,20,25\right.$ et $\left.30^{\circ} \mathrm{C}\right)$ dans des conditions de milieu et de nourriture naturelles. Parmi les caractères étudiés figurent : le taux de croissance, la taille à la maturité, la taille maximale, la durée de la phase juvénile, la durée de vie, la fécondité, le nombre de descendants par femelle, le nombre de portées et le nombre d'œufs par portée.

Les caractères démographiques des quatre populations révèlent leur adaptation à des conditions spécifiques et leur aptitude à se développer à des périodes différentes. Le faible nombre d'œufs par portée, quelle que soit l'espèce, indique une limitation des ressources exploitables.

The effect of temperature on the growth and reproduction of four species of planktonic Cladocera (Crustacea) reared with natural resources present

Keywords : growth rate, reproduction, Crustacea, Cladocera, temperature.

The growth and the reproduction of four species of Cladocera (Daphnia pulex, D. lumholtzi, Ceriodaphnia dubia et Diaphanosoma brachyurum) from a Moroccan reservoir (Lalla Takerkoust, near Marrakech) have been studied at five temperature $\left(10,15,20,25\right.$ and $\left.30^{\circ} \mathrm{C}\right)$ under natural environmental conditions (food and water quality). The characters studied are : the individual growth rate, the size of female at maturity, their maximal size, the duration of juvenile development, the duration of female life, their fecundity, the number of offsprings per female, the number of broods, the number of eggs per brood.

The demographic characters of the four populations show their adaptation to various specific environmental conditions and their aptitude for developing in the reservoir at different times. The low number of eggs per brood in all species points out a limitation in the level of available resources.

\section{Introduction}

L'étude de la structure des écosystèmes aquatiques a montré récemment que les spectres de taille des organismes composant la communauté planctonique constituaient une caractéristique essentielle (Peters 1983), en particulier pour déterminer les rela-

1. Laboratoire d'Hydrobiologie, Département de Biologie, Faculté des Sciences Semlalia, Université Cadi Ayyad, Boulevard Prince Moulay Abdellah, B.P. S 15, Marrakech, Maroc.

2. Laboratoire de Géologie Appliquée, B 123, Université Paris 6, 4, place Jussieu, F - 75252 Paris Cedex. tions trophiques. Ainsi, la production potentielle des poissons peut-elle être prédite d'après les spectres de taille des organismes planctoniques (Borgmann et al. 1984). Or, la taille et l'abondance du zooplancton dépendent essentiellement de la température et du niveau des ressources exploitables, principaux facteurs agissant, avec la prédation et la compétition, sur la croissance et la reproduction des populations. La vitesse du développement embryonnaire dépend, quant à elle, essentiellement de la température (Bottrell et al. 1976) qui agit aussi sur le développement post-embryonnaire également influencé par le facteur nutritionnel. 
Malgré la réalisation d'un nombre non négligeable de travaux concernant essentiellement les Daphnies (rev. in Threkeld 1987), les connaissances sur les caractéristiques biologiques des Cladocères planctoniques en conditions naturelles restent limitées à quelques publications (Gras \& Saint-Jean 1976, 1978, Cowgill et al. 1985, Hanazato \& Yasuno 1985).

Etant donné l'insuffisance des connaissances concernant les Cladocères récoltés dans le lac réservoir Lalla Takerkoust et l'utilité d'obtenir des données démographiques dans des conditions d'alimentation naturelle (phytoplancton), le but de ce travail est d'étudier l'influence de la température sur la croissance et la reproduction de quatre espèces de Cladocères dominantes dans la retenue : Daphnia pulex, D. lumholtzi, Ceriodaphnia dubia et Diaphanosoma brachyurum. Bosmina longirostris, autre espèce dominante dans le lac, n'a pas pu être maintenue en élevage. Cette espèce meurt 2 à 3 jours après le début des expériences à toutes les températures testées, la cause de mortalité étant probablement due à sa rétention fréquente en surface.

Les connaissances obtenues permettront d'aborder la dynamique et la production des populations de Cladocères dans les réservoirs jusqu'à présent peu étudiés.

\section{Matériel et méthodes}

\subsection{Caractéristiques générales de la retenue}

Le bassin versant de la retenue Lalla Takerkoust se caractérise par un climat méditerranéen aride à semi-aride. Les températures de l'eau restent généralement supérieures à $10^{\circ} \mathrm{C}$ et ne dépassent pas $30^{\circ} \mathrm{C}$. L'écart thermique saisonnier est important et peut atteindre jusqu'à 15 à $18^{\circ} \mathrm{C}$ entre l'été et l'hiver. C'est un lac monomictique chaud présentant une stratification estivale et une homogénéisation hivernale et automnale.

De type mésotrophe, il est caractérisé par une ceinture végétale réduite, une charge en poissons relativement importante et un phytoplancton dominé par des espèces de petite taille, en particulier par la Diatomée Cyclotella ocellata (Tableau 1).

\subsection{Méthodes d'étude}

Les Cladocères utilisés dans ces expériences proviennent de femelles récoltées dans le lac réservoir. Les expériences ont été effectuées à deux époques différentes : en avril 1990, lorsque coexistent $D$. lumholtzi, $C$. dubia et $D$. brachyurum et en janvier 1991, lors du maximum de $D$. pulex.

Les femelles de Cladocères sont suivies séparément dans des cristallisoirs en verre de $3,8 \mathrm{~cm}$ de diamètre et de $2,5 \mathrm{~cm}$ de hauteur. Les cristallisoirs, recouverts chacun par un verre de montre et contenant chacun $15 \mathrm{~mL}$ d'eau du lac sont placés dans des bains thermostatés à différentes températures : $10,15,20$ et $30^{\circ} \mathrm{C}$. Afin d'éliminer tout risque éventuel d'interférence spécifique avec la durée ou l'intensité d'éclairement, les expériences sont réalisées à l'obscurité, condition qui ne perturbe ni la nutrition ni la reproduction parthénogénétique. $\mathrm{Par}$ nécessité pratique, les animaux ne sont soumis qu'à un éclairement très temporaire (quelques minutes) au moment de l'examen des élevages.

Le milieu d'élevage, renouvelé chaque jour est composé par de l'eau du lac contenant le phytoplancton à sa concentration naturelle. La composition de ce phytoplancton varie selon les époques où les expériences ont été réalisées (Tableau 1). Le phytoplancton d'avril 1990, utilisé pour alimenter D. lumholtzi, D. brachyurum, C. dubia, est dominé par la Diatomée centrique Cyclotella ocellata qui représente $95 \%$ du biovolume total. Or, cette espèce est bien consommée par tous les stades de tous les Cladocères expérimentés (Cherifi 1992). Il en va de même vraisemblablement de Carteria sp., flagellé de petite taille, nettement moins abondant $(2,8 \%$ du total). En admettant que la teneur en carbone représente $50 \%$ du poids sec, lui-même égal à $40 \%$ du biovolume (Reynolds, 1984), la teneur en carbone de ce phytoplancton peut être estimée à environ $0,3 \mathrm{mg} \mathrm{C} / \mathrm{L}$. En revanche, le phytoplancton de janvier 1991, utilisé pour nourrir $D$. pulex, est bien plus pauvre en algues consommables par cette espèce : petits Pediastrum, vraisemblablement Schroederia et Cyclotella. Cette dernière espèce représente $44 \%$ du biovolume total qui est près de $\mathbf{3 0}$ fois inférieur à celui des ressources disponibles en avril 1990 (Tableau 1).

Les expériences sont réalisées immédiatement après récolte des animaux dans le milieu naturel. Avant le début de chaque expérience, les femelles ovigères sont d'abord maintenues en élevage aux températures choisies jusqu'à libération des nouveaux nés qu'elles portaient. Une dizaine de jeunes ainsi acclimatés à la température d'expérience sont 
Tableau 1. Composition du phytoplancton (en nombre de cellules par litre) fourni comme nourriture aux quatre espèces de Cladocères expérimentées. $\left(^{*}=\right.$ algues considérées comme consommables) (Cherifi, comm. pers.).

Table 1. Phytoplancton composition (in number of cells per liter) used for feeding the four species of Cladocera $(*$ = edible algae) (Cherifi, pers. comm.).

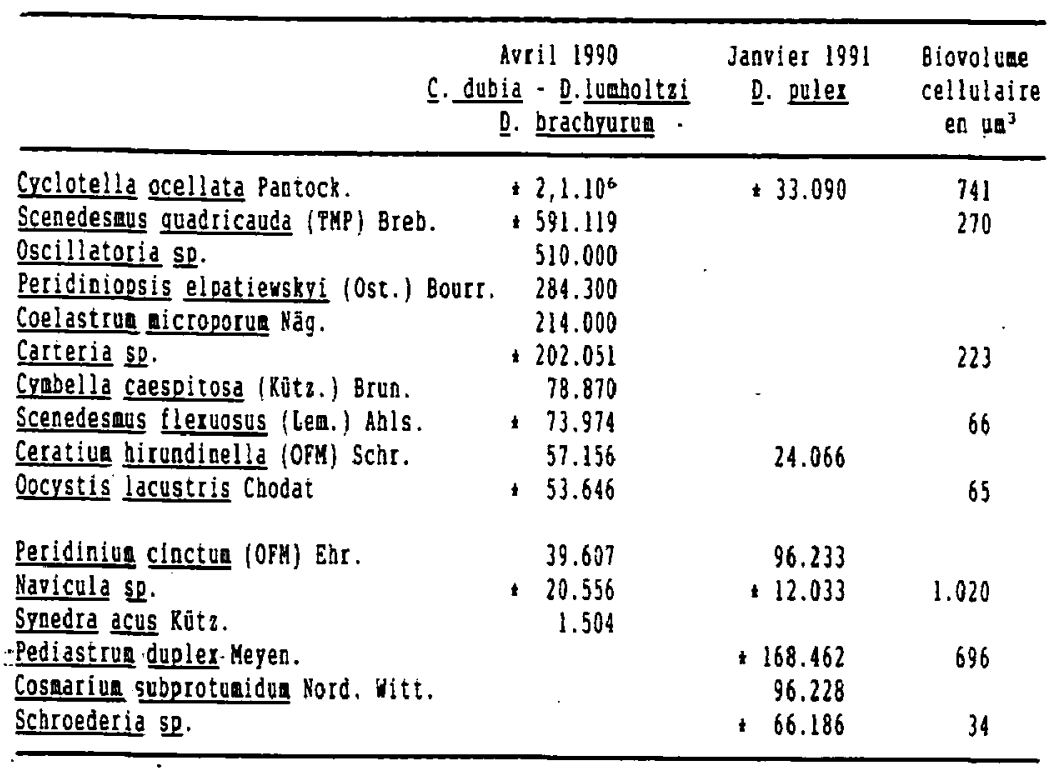

placés séparément dans des cristallisoirs et suivis jusqu'à donner des femelles ovigères qui libèrent à leur tour des nouveaux-nés. Ces derniers sont ensuite retirés et les mères sont transférées dans un milieu neuf et ainsi de suite jusqu'à la mort des individus. La mortalité observée est notée pour chaque espèce et à chaque température expérimentale. L'observation des élevages a lieu deux fois par jour, ce qui permet une évaluation suffisamment précise pour la plupart des variables étudiées, à l'exception de la durée de développement embryonnaire.

\section{Résultats}

Nous avons déterminé pour chaque espèce et pour chaque température :

- le taux de croissance juvénile,

- la durée de développement embryonnaire, De, séparant la ponte des œufs de la libération des jeunes,

- la durée de développement juvénile, $\mathrm{Dj}$, allant de l'expulsion de la jeune femelle à sa première ponte,

- la durée de vie des femelles et leur taux de survie,

- la fécondité par stade (âge des femelles),

- le nombre moyen de jeunes poduits par une femelle durant sa vie,
- le taux de mortalité embryonnaire,

- le nombre de portées par femelle et le nombre de jeunes par portées.

\section{Daphnia lumholtzi Sars 1886}

$D$. lumholtzi ne survit qu'entre 15 et $25^{\circ} \mathrm{C}$ : dans toutes les expériences réalisées à 10 et $30^{\circ} \mathrm{C}$, aucune femelle ne vit plus de 2 à 3 jours. Les jeunes issus de ces femelles meurent quelques heures après leur naissance à $30^{\circ} \mathrm{C}$ alors qu'à $10^{\circ} \mathrm{C}$, les jeunes subsistent pendant une semaine sans jamais atteindre la maturité. Entre 15 et $25^{\circ} \mathrm{C}$, la survie des femelles est identique aux trois températures (Fig. 1).

Sa croissance est optimale à $20^{\circ} \mathrm{C}, 0,43 \mathrm{~mm} /$ jour (Fig. 2 et Tableau 2). Le taux de croissance varie peu entre 20 et $25^{\circ} \mathrm{C}$. La taille maximale $(1,80$ à 2,04 $\mathrm{mm})$, atteinte dès la première reproduction est semblable à celle de $D$. pulex et supérieure à celle d'autres populations (1,69 mm selon Lynch 1980).

La durée du développement juvénile proche de 5 jours à des températures élevées s'accroît nettement lorsque la température s'abaisse à $15^{\circ} \mathrm{C}$, alors que la durée de vie moyenne d'une femelle ne varie pas significativement et se situe entre 18 et 20 jours (Tableau 2).

La fécondité des premiers stades $(\mathrm{C} 4, \mathrm{C} 5)$ est la plus élevée et diminue ensuite. Cette régression est plus marquée à $15^{\circ} \mathrm{C}$, où la fécondité reste très faible (Fig. 3).

De même, le nombre total de jeunes produits par une femelle pendant sa vie, quoique très variable, est plus important à $20^{\circ} \mathrm{C}$ où il est en moyenne de 15 (maximum = 40) (Fig. 4). Encore élevé et proche de 10 à $25^{\circ} \mathrm{C}$, le nombre de descendants produits par femelle diminue significativement à $15^{\circ} \mathrm{C}$ (Tableau 2). Ceci est dû à une réduction du nombre de portées alors que le nombre d'œufs par portée varie peu avec la température (Fig. 5).

Le taux de mortalité embryonnaire reste faible et toujours inférieur à $7 \%$.

\section{Daphnia pulex Leydig 1860}

D. duplex ne survit pas à $30^{\circ} \mathrm{C}$ : dans tous les essais réalisés à cette température, les jeunes daphnies meurent 1 à 2 jours au maximum après leur naissance et aucune des femelles ovigères acclimatées ne survit au delà de 24 heures. A $25^{\circ} \mathrm{C}$, le taux de survie est encore inférieur à ceux observés aux températures plus basses (Fig. 1). 
Survie (\%)
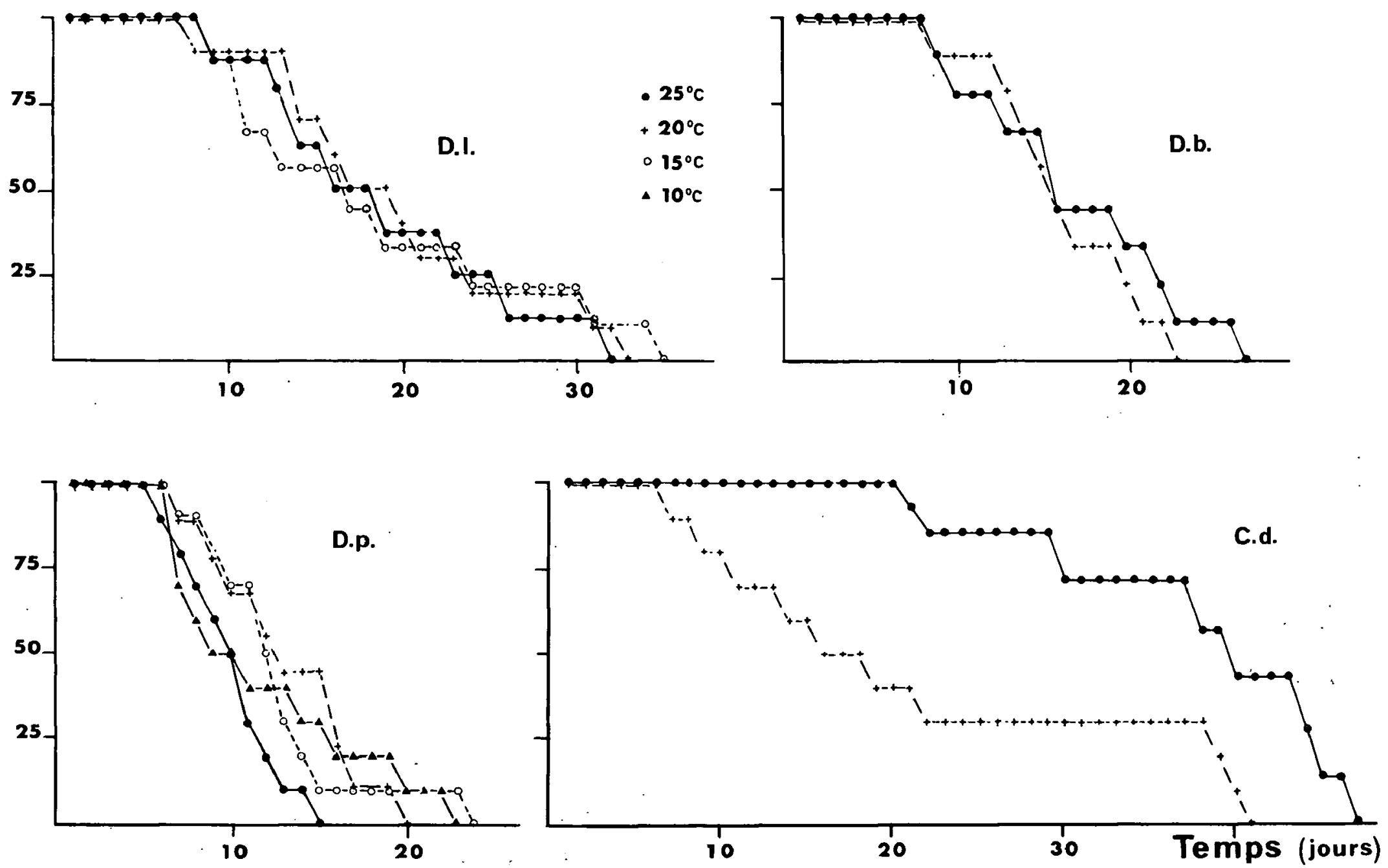

Fig. 1. Courbes de survie des quatre espèces de Cladocères à différentes températures. D.1. : Daphnia lumholtiz, D.p. : D. pulex, D.b. : Diaphanosoma brachyurum, C.d. : Ceriodaphnia dubia.

Fig. 1. Survival in the four cladoceran species at various temperatures. D.1. : Daphnia lumholtzi, D.p. : D. pulex, D.b. : Diaphanosoma brachyurum, C.d. : Ceriodaphnia dubia.

Le taux de croissance de $D$. pulex augmente avec la température. Sa croissance optimale, 0,62 $\mathrm{mm} /$ jour, est obtenue à $25^{\circ} \mathrm{C}$ (Tableau 2).

Le développement juvénile $(\mathrm{Dj})$ demeure très long à $10^{\circ} \mathrm{C}$ et à $15^{\circ} \mathrm{C}$, alors qu'à 20 et $25^{\circ} \mathrm{C}$, les jeunes atteignent rapidement la maturité sexuelle. En effet, les femelles de $D$. pulex atteignent leur taille maximale $(1,80$ à $2,02 \mathrm{~mm})$ entre le 4ème et le 5ème jours à $25^{\circ} \mathrm{C}$. Après les premières pontes, la croissance est plus lente et peut même s'arrêter (Fig. 2).

La durée de vie moyenne de $D$. pulex est relativement stable ( 12 à 13 jours) entre 10 et $20^{\circ} \mathrm{C}$ puis baisse à $25^{\circ} \mathrm{C}$ (Tableau 2).

La fécondité est maximale dès le premier stade mature (C4) et diminue ensuite, particulièrement à $15^{\circ} \mathrm{C}$ (Fig. 3).
Le nombre total moyen de descendants par femelle est plus élevé à 20 et $25^{\circ} \mathrm{C}(8,8$ et 8,9 jeunes par femelle) qu'à 10 et $15^{\circ} \mathrm{C}$ (respectivement de 0,7 à 5,2 jeunes par femelle) mais, de 15 à $25^{\circ} \mathrm{C}$, les écarts ne sont pas significatifs. Le nombre de portées par femelle et le nombre d'œufs par portée ne varient pas non plus significativement (Tableau 2 et Fig. 5).

Le taux dè mortalité des embryons est maximal à $10^{\circ} \mathrm{C}(22,2 \%)$ et minimal à $15^{\circ} \mathrm{C}(3,7 \%)$ puis augmente à nouveau avec la température (Tableau 2 ).

\section{Ceriodaphnia dubia Richard 1894}

C. dubia ne survit qu'à 20 et $25^{\circ} \mathrm{C}$ : les femelles ovigères acclimatées aux autres températures meurent 2 à 3 jours après le début des expériences. 

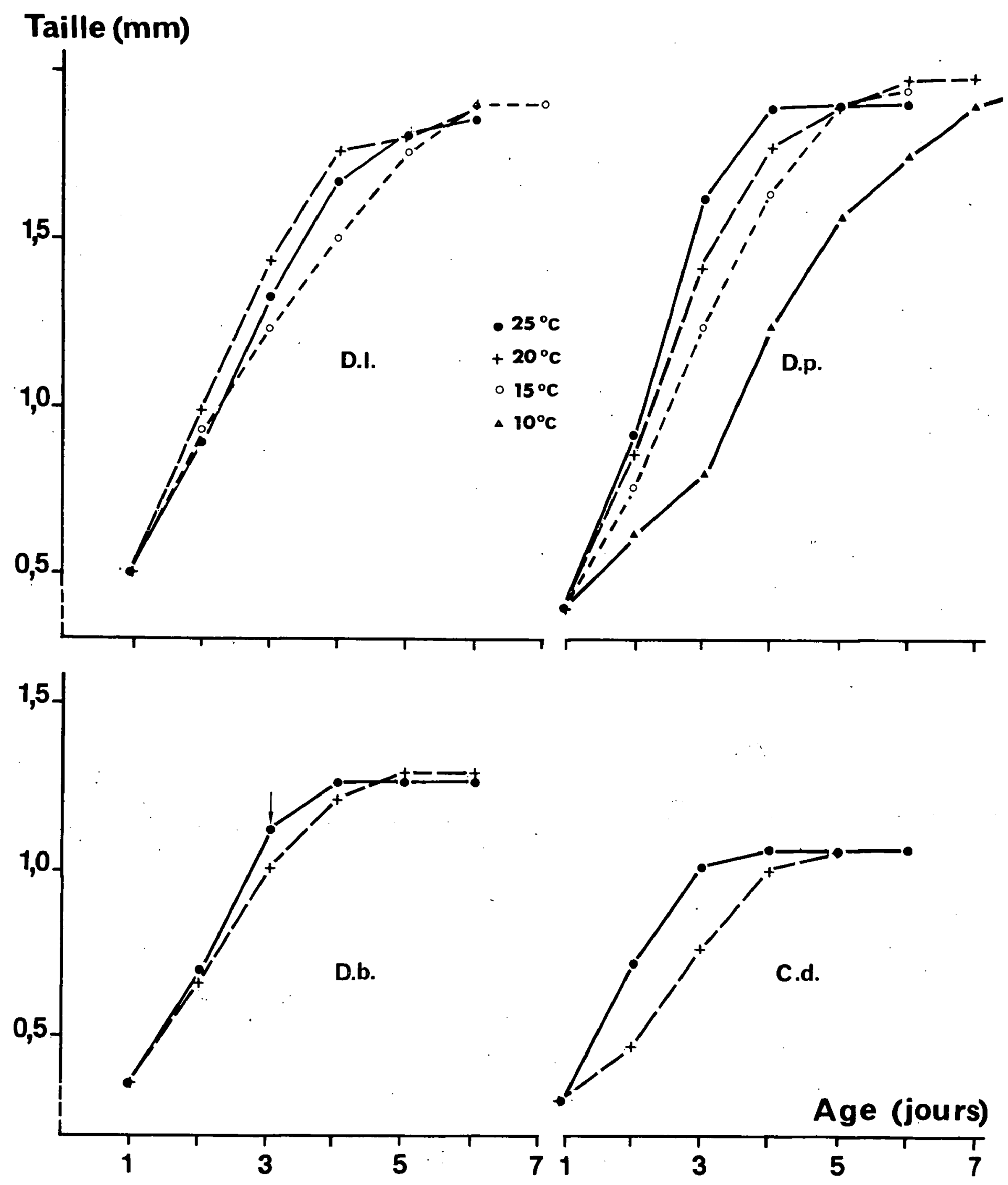

Fig. 2. Courbes de croissance des quatre espèces de Cladocères à différentes températures. Mêmes symboles que fig. 1 .

Fig. 2. Growth curves of four cladoceran species at various temperatures. Symbols as in Fig. 1.

De même, les jeunes Ceriodaphnia libérées par certaines femelles meurent quelques heures, au maximum 24 heures, après leur naissance, sauf à $15^{\circ} \mathrm{C}$ où elles vivent environ 10 jours. Leur survie est nettement meilleure à $25^{\circ} \mathrm{C}$ (Fig.1).
Le taux de croissance optimal est observé à $25^{\circ} \mathrm{C}$. La taille maximale $(1,1 \mathrm{à} 1,13 \mathrm{~mm})$ est atteinte en $4\left(25^{\circ} \mathrm{C}\right)$ à 5 jours $\left(20^{\circ} \mathrm{C}\right)$ (Fig. 2).

Les premières femelles ovigères apparaissent dès le 3ème ou le 4ème jour à $25^{\circ} \mathrm{C}$ alors qu'à $20^{\circ} \mathrm{C}$, 
Tableau 2. Caractères démographiques de quatre espèces de Cladocères à différentes températures : taux de croissance juvénile, taille à la maturité, temps de développements embryonnaire (De) et juvénile ( $\mathrm{Dj}$, de la naissance à la première ponte), durée de vie, nombre de jeunes produits par une femelle durant sa vie, taux de mortalité embryonnaire, nombre de portées par femelle et nombre d'œufs par portée. Le nombre de femelles (ou de portées) observées est indiqué entre parenthèses. ${ }^{*}=$ écart significatif à $\mathrm{p}<$ 0,05 avec la valeur suivante ${ }^{* *}=$ id. à $\mathrm{p}<0,01$.

Table 2. Demographic characters of four species of Cladocera at various temperatures : rate of individual growth, size at maturity, duration of embryonic development (De), duration of juvenil growth $(\mathrm{Dj}$, from the egg hatching to the first egg laying), duration of life, total number of youngs produced per female, rate of embryonic mortality, number of broods per female and number of eggs per brood. The number of observed females (or broods) is noted in brackets. ${ }^{*}=$ significant difference at $p<0,05$ with the following value $;^{* *}=$ id. at $p<0,01$.

\begin{tabular}{|c|c|c|c|c|c|}
\hline Espèces & $\begin{array}{l}\text { tures en }{ }^{\circ} \mathrm{C} \\
\text { Variables }\end{array}$ & 10 & 15 & 20 & 25 \\
\hline Daphnia pules & $\begin{array}{l}\text { Taus de crois. } \\
\text { Taille à maturité } \\
\text { De } \\
\text { Dj } \\
\text { Durée de vie } \\
N \text { jeunes/fem. } \\
\text { mort. embryon. } \\
N \text { portées/fem. } \\
N \text { oeufs/portée }\end{array}$ & $\begin{array}{c}0,29 \\
1,92 \pm 0,02 \quad(3 \\
= \\
8,0 \pm 1,7(3) \\
12,3 \pm 6,6(10) \\
0,7 \pm 1,1 \quad(10)= \\
22,28 \\
- \\
\end{array}$ & $\begin{array}{c}0,42 \\
1,93 \pm 0,07 \\
2,2 \pm 1,0(11) \\
6,7 \pm 1,8(8) \pm \pm \\
12,5 \pm 4,8(10) \\
5,2 \pm 5,3(10) \\
3,78 \\
1,5 \pm 0,8(8) \\
4,5 \pm 1,3(12)\end{array}$ & $\begin{array}{c}0,51 \\
1,97 \pm 0,03 \\
1,4 \pm 0,2 \\
4,3 \pm 1,5(8) \\
13,3 \pm 4,2(9) \\
8,9 \pm 8,2(9) \\
10,18 \\
3,0 \pm 1,8(8) \\
3,7 \pm 1,1(24)\end{array}$ & $\begin{array}{c}0,62 \\
1,91 \pm 0,06 . \\
1,2 \pm 0,2 \\
3,7 \pm 0,8(10) \\
10,2 \pm 3,0(10) \\
8,8 \pm 5,3(10) \\
16,2 \\
2,7 \pm 1,1(10) \\
3,9 \pm 1,1(27)\end{array}$ \\
\hline D. Iunholtzi & $\begin{array}{l}\text { Taux de crois. } \\
\text { Taille à aturité } \\
\quad \text { De } \\
\quad \text { Dj } \\
\text { Durée de vie } \\
N \text { jeunes/fem. } \\
\text { mort. embryon. } \\
\text { N portées/fen. } \\
N \text { oeufs/portée }\end{array}$ & $\begin{array}{l}. \\
\dot{-} \\
. \\
- \\
\dot{-} \\
.\end{array}$ & $\begin{array}{c}0 ; 31 \\
1,92 \pm 0,05 \\
1,9 \pm 0,9(18) \pm \pm \\
8,7 \pm 2,3(7) \pm * \\
18,9 \pm 9,3(9) \\
4,4 \pm 6,3(9) \pm \\
0 \\
2,6 \pm 2,4(7) \pm \\
2,2 \pm 1,1(18)\end{array}$ & $\begin{array}{c}0,43 \\
1,90 \pm 0,09 \\
1,1 \pm 0,2(64) \\
4,7 \pm 1,1(10) \\
19,8 \pm 7,8(10) \\
15,0 \pm 11,3(10) \\
2,68 \\
6,4 \pm 4,0(10) \\
2,4 \pm 1,2(64)\end{array}$ & $\begin{array}{c}0,40 . \\
1,87 \pm 0,07 \\
1,1 \pm 0,3(37) \\
5,3 \pm 1,6(8) \\
18,0 \pm 7,7(9) \\
9,0 \pm 7,9(9) \\
3,68 \\
4,9 \pm 3,9(8) \\
2,2 \pm 1,2(39)\end{array}$ \\
\hline $\begin{array}{l}\text { Diaphanosoma } \\
\text { brachyurum }\end{array}$ & 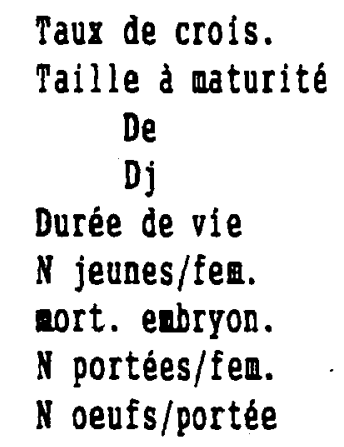 & $\begin{array}{l}- \\
- \\
- \\
- \\
- \\
- \\
-\end{array}$ & $\begin{array}{c}- \\
- \\
- \\
- \\
8(10) \\
0 \\
- \\
- \\
-\end{array}$ & $\begin{array}{c}0,34 \\
1,27 \pm 0,03 \\
1,1 \pm 0,3(36) \\
4,6 \pm 2,4(9) \\
16,4 \pm 4,4(9) \\
13,1 \pm 6,0(9) \\
4,18 \\
4,2 \pm 1,9(9) \\
3,2 \pm 1,1(38)\end{array}$ & $\begin{array}{c}0,38 \\
1,24 \pm 0,03 \\
1,2 \pm 0,4(46) \\
3,1 \pm 0,6(9) \\
17,3 \pm 6,1(9) \\
18,6 \pm 10,0(9) \\
2,98 \\
5,4 \pm 2,7(9) \\
3,5 \pm 1,1(49)\end{array}$ \\
\hline$\frac{\text { Ceriodaphnia }}{\underline{\text { dubia }}}$ & $\begin{array}{c}\text { Taur de crois. } \\
\text { Taille à adurité } \\
\text { De } \\
\text { Dj } \\
\text { Durée de vie } \\
\mathbb{N} \text { jeunes/fem. } \\
\text { wort. eabryon. } \\
\mathbb{N} \text { portées/fem. } \\
\mathbb{N} \text { oeufs/portée }\end{array}$ & $\begin{array}{l}- \\
- \\
- \\
- \\
- \\
- \\
- \\
-\end{array}$ & $\begin{array}{c}- \\
- \\
- \\
- \\
10(10) \\
0 \\
- \\
- \\
-\end{array}$ & $\begin{array}{c}0,24 \\
1,06 \pm 0,06 \\
1,4 \pm 0,5(45) \pm \pm \\
4,8 \pm 1,0(10) \\
21,8 \pm 13,3(10) \pm \\
11,5 \pm 12,8(10) \pm \pm \\
1,78 \\
4,5 \pm 4,1(10) \pm \pm \\
2,6 \pm 1,2(45)\end{array}$ & $\begin{aligned} 0,35 \\
1,05 \pm 0,06 \\
1,2 \pm 0,3(98) \\
4,4 \pm 2,0(7) \\
38,0 \pm 9,5(7) \\
36,9 \pm 12,4(7) \\
\\
0 \\
14,0 \pm 4,3(7) \\
2,6 \pm 1,3(98\end{aligned}$ \\
\hline
\end{tabular}



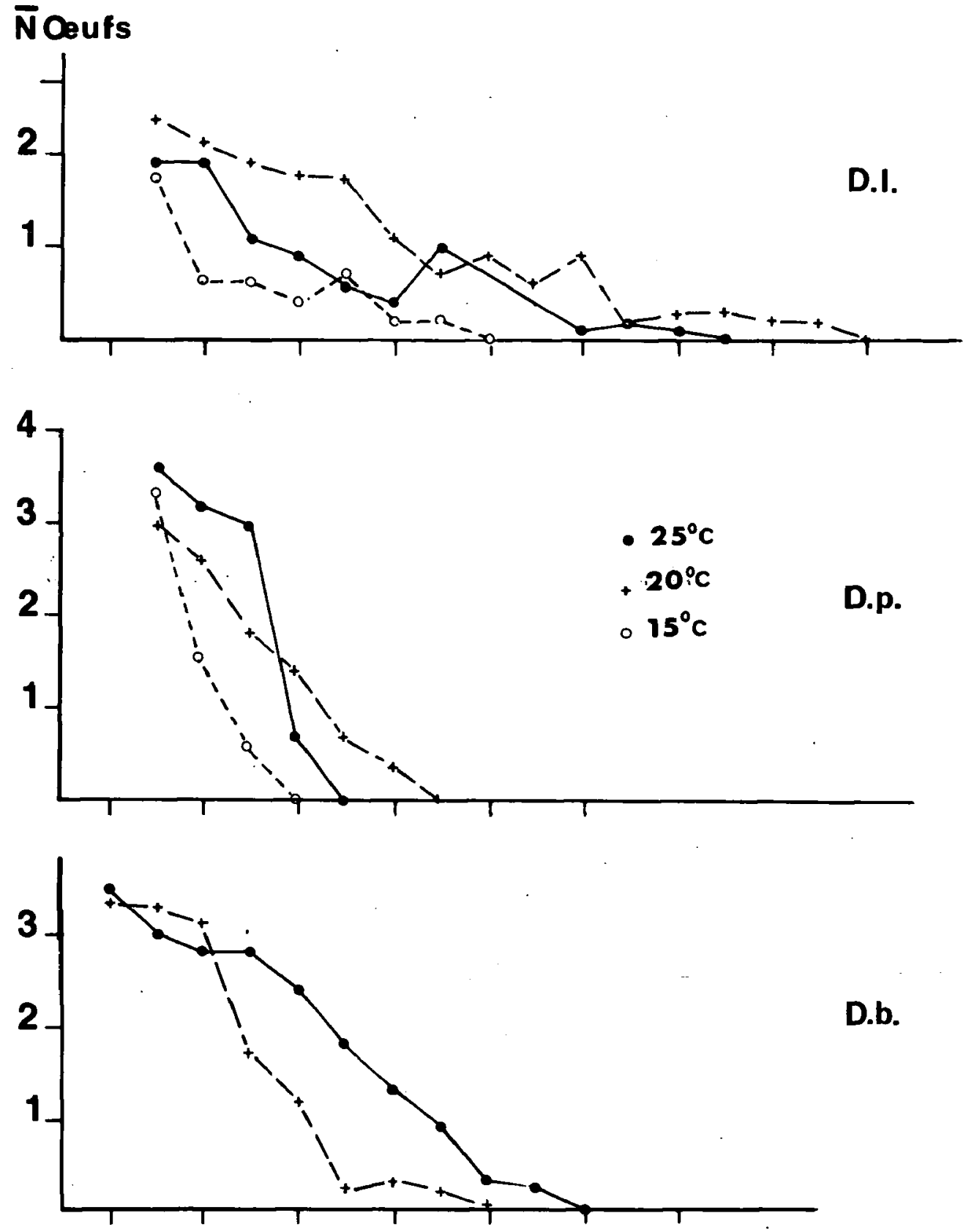

D.b.

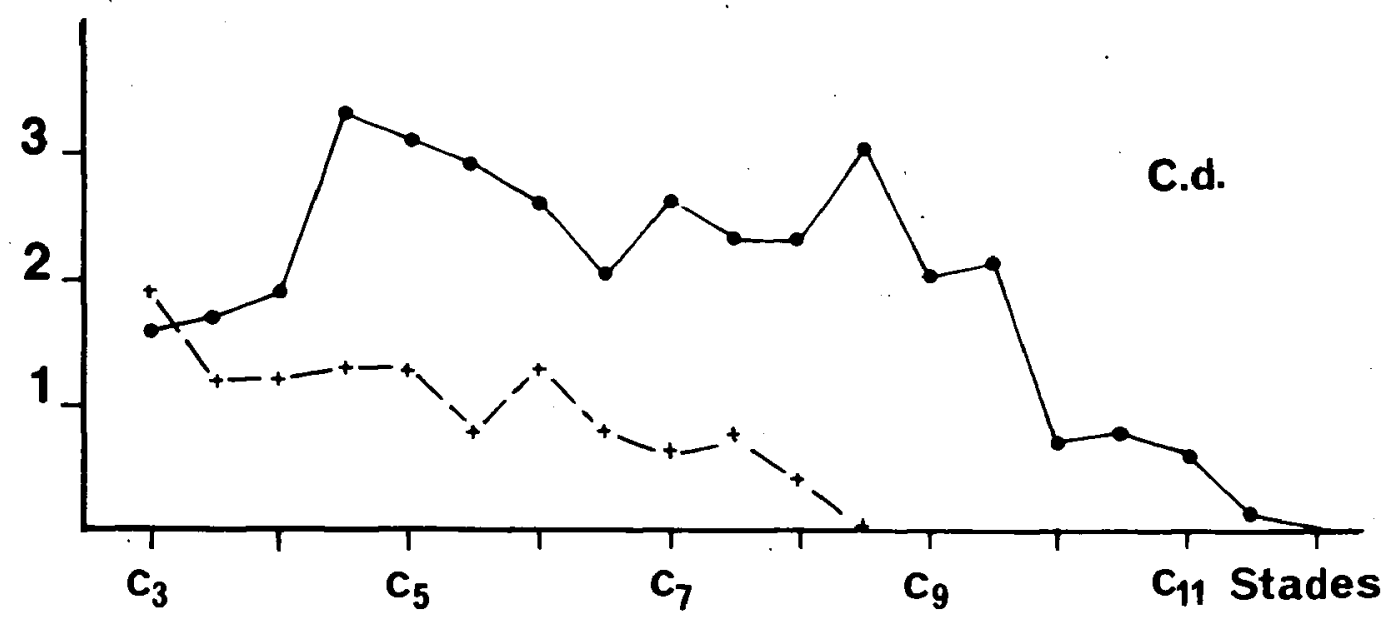

Fig. 3. Variations du nombre d'œufs pondus à chaque stade pour les quatre espèces, à différentes températures. Mêmes symboles qu'à la figure 1.

Fig. 3. Variations of the eggs number per instar in the four species, at various temperature. Symbols as in Fig. 1. 
elles apparaissent 1 à 2 jours plus tard. Néanmoins, du fait d'une forte variabilité à $25^{\circ} \mathrm{C}$, la durée moyenne du développement juvénile $(\mathrm{Dj})$ ne diffère pas significativement à 20 et à $25^{\circ} \mathrm{C}$ (Tableau 2).

La durée de vie moyenne double approximativement pour une augmentation de la température de $5^{\circ} \mathrm{C}$ (Tableau 2).

A $25^{\circ} \mathrm{C}$, la fécondité augmente jusqu'au stade $\mathrm{C} 6$, présente ensuite un plateau et un second maximum au stade $\mathrm{C} 14$ puis régresse. $\mathrm{A} 20^{\circ} \mathrm{C}$, la fécondité est nettement plus faible et diminue dès le stade $\mathrm{C} 3$ (Fig. 3).

Le nombre moyen de jeunes produits par une femelle durant sa vie est plus de trois fois supérieur à 25 qu'à $20^{\circ} \mathrm{C}$ (Fig. 4 et 6 , Tableau 2). Cette augmentation de la fécondité provient du triplement du nombre de portées alors que le nombre d'œufs par portée reste constant (Fig. 5).

Le taux de mortalité embryonnaire est très faible aux températures testées : $1,7 \%$ à $20^{\circ} \mathrm{C}$ et nul à $25^{\circ} \mathrm{C}$.

\section{Diaphanosoma brachyurum Liévin 1848}

D. brachyurum ne survit qu'à 20 et $25^{\circ} \mathrm{C}$ : comme C. dubia, les femelles ovigères testées à 10,15 et à $30^{\circ} \mathrm{C}$ meurent 4 à 5 jours après le début des expériences.
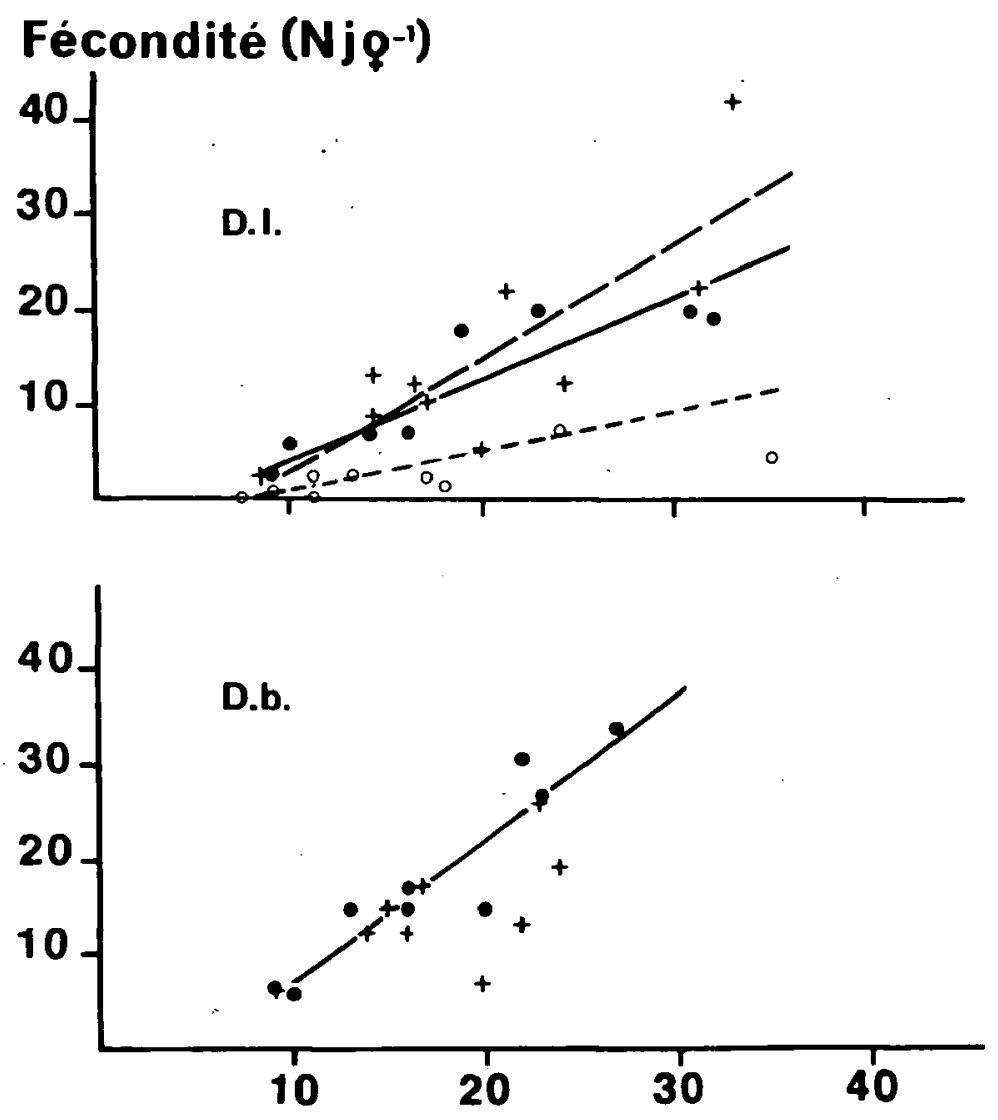

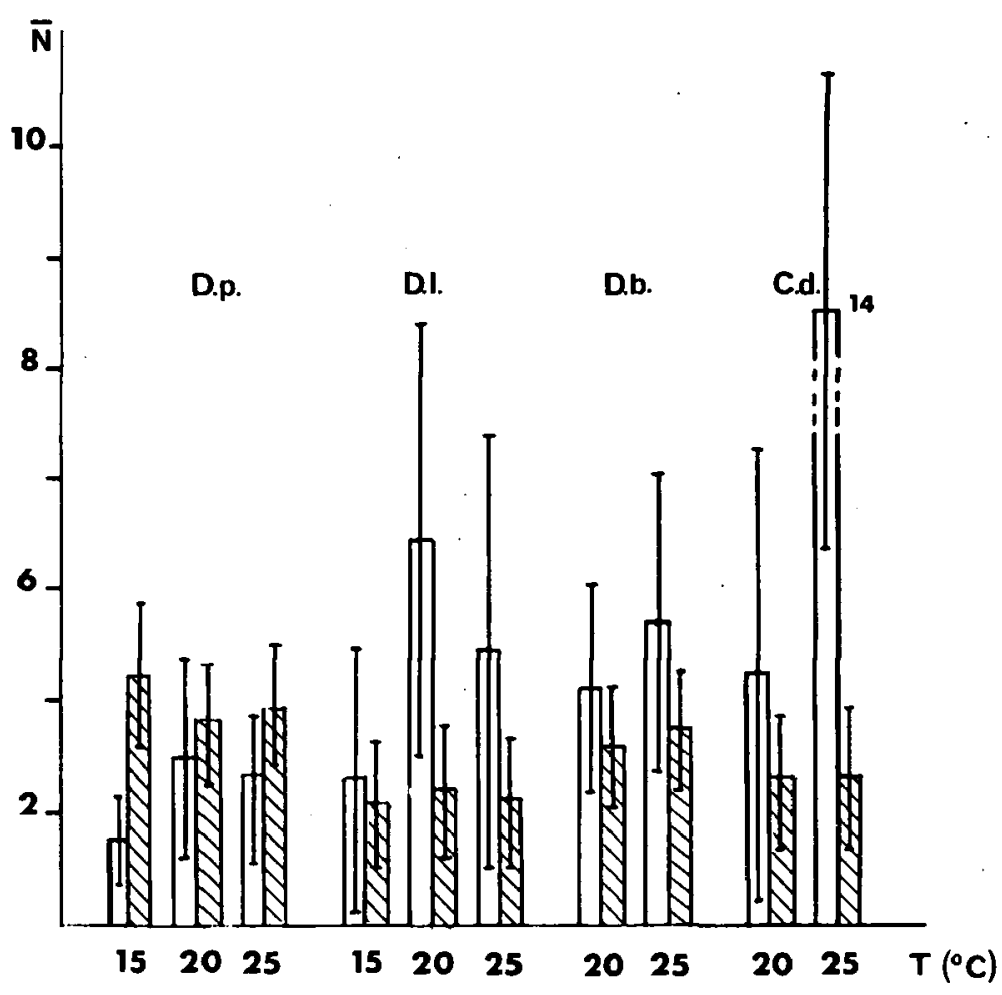

Fig. 5. Nombre moyen de portées par femelle (en clair) et nombre moyen d'œufs par portée (en hachuré) pour les quatre espèces étudiées à différentes températures. Mêmes symboles que Fig. 1.

Fig. 5. Mean number of broods per female (clear) and mean number of eggs per brood (hachured) in the four species cultured at various temperatures. Symbols as in Fig. 1.
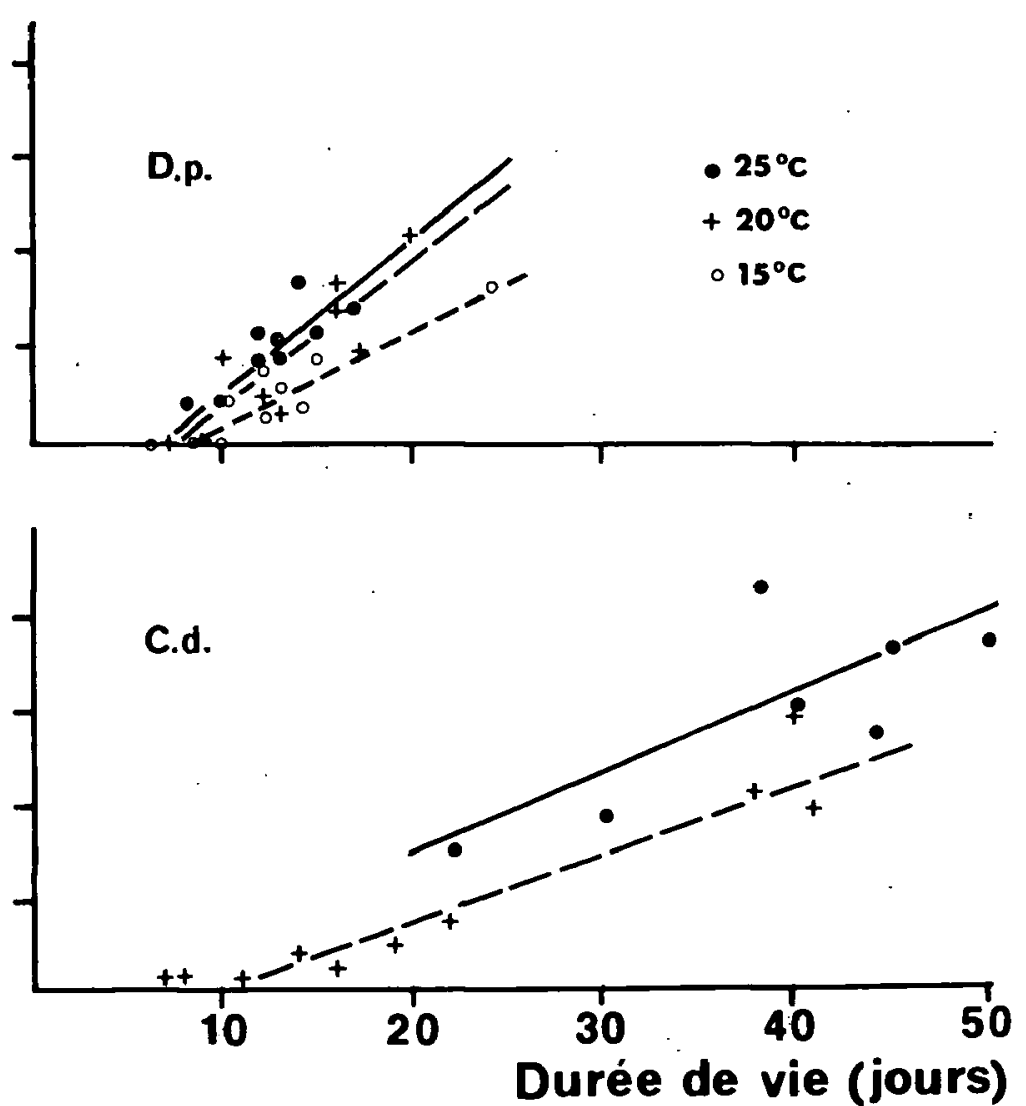

Fig. 4. Nombre total de descendants produits par chaque femelle en relation avec sa durée de vie, à différentes températures. Mêmes symboles que Fig. 1.

Fig. 4. Total number of offsprings produced per each female in relation to her life time, at various temperatures. Symbols as in Fig. 1 . 
A $15^{\circ} \mathrm{C}$, les jeunes libérés par des femelles ovigères survivent 7 à 10 jours et une seule femelle a pu produire une unique portée de 3 embryons. Contrairement à celles de $C$. dubia, les courbes de survie de D. brachyurum sont similaires à 20 et $25^{\circ} \mathrm{C}$ (Fig. 1).

Le taux de croissance, la durée de vie et le temps de développement juvénile ne sont pas significativement différents à 20 et à $25^{\circ} \mathrm{C}$ (Tableau 2, Fig. 2). Toutefois, les Diaphanosoma acquièrent leur maturité en moins de quatre jours à $25^{\circ} \mathrm{C}$ (les premières femelles ovigères apparaissent entre le 3ème et le 4ème jour) alors que l'acquisition de la maturité nécessite un jour de plus à $20^{\circ} \mathrm{C}$. La taille maximale atteinte dès le 5ème jour est de 1,2 à $1,3 \mathrm{~mm}$ : elle est légèrement supérieure à celle connue par ailleurs (1,13 mm selon Lynch 1980).

La fécondité diminue avec l'âge des femelles, plus rapidement à $20^{\circ} \mathrm{C}$ qu'à $25^{\circ} \mathrm{C}$, particulièrement à partir du stade C5 (Fig. 3).

Bien que le nombre moyen de jeunes et le nombre de portées par femelle soient supérieurs à $25^{\circ} \mathrm{C}$ qu'à $20^{\circ} \mathrm{C}$ et la mortalité embryonnaire faible dans les deux cas, les différences ne sont pas significatives (Tableau 2). Enfin, le nombre d'œufs pondus pas une femelle est significativement corrélé à sa longévité à $25^{\circ} \mathrm{C}$ mais non à $20^{\circ} \mathrm{C}$ (Fig. 4).

\section{Discussion et conclusion}

Les résultats obtenus permettent de déterminer les seuils de tolérance à la température de ces quatre espèces. Trente degrés sont une température létale pour toutes les espèces qui ne diffèrent donc que par leur seuil inférieur de létalité.

C. dubia et $D$. brachyurum sont les moins tolérantes, n'acceptant de vivre et de se reproduire que de 20 à $25^{\circ} \mathrm{C}$. A l'inverse, $D$. pulex s'avère la plus tolérante, vivant aux températures les plus basses (10 et $15^{\circ} \mathrm{C}$ ), son seuil de reproduction se situant vers $10^{\circ} \mathrm{C}$. D. lumholtzi occupe une position intermédiaire avec un seuil de létalité à $10^{\circ} \mathrm{C}$ (Tableau 2).

L'interprétation des observations et la comparaison des résultats est particulièrement valable pour les trois espèces. $D$. lumholtzi, $C$. dubia et $D$. brachyurum, auxquelles il a été fourni une alimentation naturelle similaire en qualité et en quantité (dominance des Cyclotella). Connaissant la température et la taille des femelles, leur taux de filtration peut être estimé à partir des formules de Moure- latos et Lacroix (1990) et Mourelatos et al. (1992). Dans l'intervalle de température 20 à $25^{\circ} \mathrm{C}$, ce taux varie de 18 à $30 \mathrm{~mL}$ par individu et par jour pour des Ceriodaphnia de $1 \mathrm{~mm}$ et de 20 à 40 ou de 40 à $80 \mathrm{~mL}$ (selon l'équation utilişée) pour des Daphnies de $2 \mathrm{~mm}$, alors qu'il ne varie que de 4 à $8,8 \mathrm{~mL}$ pour des Diaphanosoma. Ce calcul montre que, à l'exception de ces derniers, les Cladocères filtrent en un jour la totalité du volume expérimental (15 $\mathrm{mL}$ ) et que la ration ingérée est limitée à la quantité de phytoplancton incluse dans ce volume (soit environ $5 \mu \mathrm{g} \mathrm{C)}$. Seule, $D$. brachyrum ne filtrerait qu'une partie du volume disponible et ne consommerait "qu'une part $(1 / 4$ ou $1 / 2)$ du phytoplancton à sa disposition.

Dans ces conditions, l'optimum de croissance et de reproduction s'observe à $20^{\circ} \mathrm{C}$ pour $D$. lumholtzi alors que les deux dernières espèces ont une durée de vie plus longue et une reproduction optimale à $25^{\circ} \mathrm{C}$. Un effet négatif des températures élevées a été aussi observé par Pace et al. (1984) chez D. parvula. La durée du développement juvénile de $D$. lumholtzi à $25^{\circ} \mathrm{C}$ est semblable à celle observée par Gras \& Saint-Jean (1978).

Herzig (1984), Hanazato \& Yasuno (1987) ont constaté que le taux de croissance et le taux de développement juvénile de $D$. brachyurum sont reliés positivement à la température dans un large intervalle de valeurs. Néanmoins, Herzig observe une nette différence entre les générations de printemps et celles d'été. Le développement post-embryonnaire de ces dernières (vraisemblablement plus proches de celui des populations marocaines) ne s'effectue avec succès qu'aux températures supérieures à $15^{\circ} \mathrm{C}$, ce qui est le cas de la population du Lalla Takerkoust. Les durées de $\mathrm{Dj}$ de celle-ci sont très proches de celles observées par Herzig (1984), Gras \& Saint-Jean (1978, sur une autre espèce du même genre) et proche du minimum calculé par Romanovsky (1984) pour une population naturelle.

Dans l'intervalle $20-25^{\circ} \mathrm{C}$, la température influence assez peu la croissance et la reproduction de $D$. brachyurum alors qu'elle affecte notablement la durée de vie et la fécondité de $C$. $d u b i a$. Il est à noter que la longévité et la fécondité de celle-ci sont supérieures à $25^{\circ} \mathrm{C}$ alors que l'inverse est observé chez des populations nord-américaines plus prolifiques et plus longévives car élevées au laboratoire avec des cultures d'algues (Cowgill et al. 1985). 
La population de $C$. dubia du réservoir Lalla Takerkoust est nettement mieux adaptée à la température de $25^{\circ} \mathrm{C}$ que $D$. brachyurum et les deux espèces du genre Daphnia ainsi que l'a observé Lynch (1978). Ainsi, la fécondité de C. dubia à $25^{\circ} \mathrm{C}$ est la seule qui augmente avec l'âge (le stade) des femelles puis se stabilise avant de décroître aux derniers stades (Fig. 3). Cette espèce semble adopter, même à ce niveau de ressources peu élevé, une stratégie du type $r$.

La croissance de $D$. pulex est fonction de la température mais celle-ci n'affecte pas sa durée de vie et assez peu le nombre de descendants produits par une femelle (Fig. 6). Comparée à ceux des trois autres espèces, ces deux paramètres ont les plus faibles valeurs chez $D$. pulex tant à 20 qu'à $25^{\circ} \mathrm{C}$. La taille maximale, la durée de vie et la fécondité de cette population apparaissent très réduites par rapport à celles habituellement données dans la littérature (Lynch 1980, 1989, Mc Cauley et al. 1990). En outre, la décroissance de la fécondité à partir du premier stade mature est nettement plus rapide chez cette espèce (Fig. 3). Les causes en sont à rechercher dans la faiblesse des ressources nutritives disponibles pour cette population (environ 30 fois inférieures à celles fournies aux trois autres espèces). Cette capacité à se reproduire en présence de ressources algale limitées implique la mise en œuvre d'une stratégie de type K par D. pulex (Schwartz 1984).

Les caractères démographiques des quatre espèces de Cladocères étudiés au laboratoire en conditions de milieu et d'alimentation naturels diffèrent nettement. Ils reflètent l'aptitude de chaque espèce à se développer dans le lac réservoir à des époques différentes : $C$. dubia et $D$. brachyurum en période chaude, $D$. pulex en période froide alors que la concurrence des autres espèces est faible, et $D$. lumholtzi aux températures moyennes.

Il est à noter que l'influence de la température sur la fécondité se traduit par une variation significative du nombre de portées, quelle que soit l'espèce considérée. En revanche, le nombre d'œufs par portée ne varie pas significativement et reste assez faible (2,2 à 4,5 en moyenne) ce qui est l'indice d'une limitation des ressources disponibles (Taylor 1985).

Enfin, toutes les espèces expérimentées investissent leur énergie d'abord dans la croissance, qui s'arrête à la maturité, puis dans la reproduction. Cette

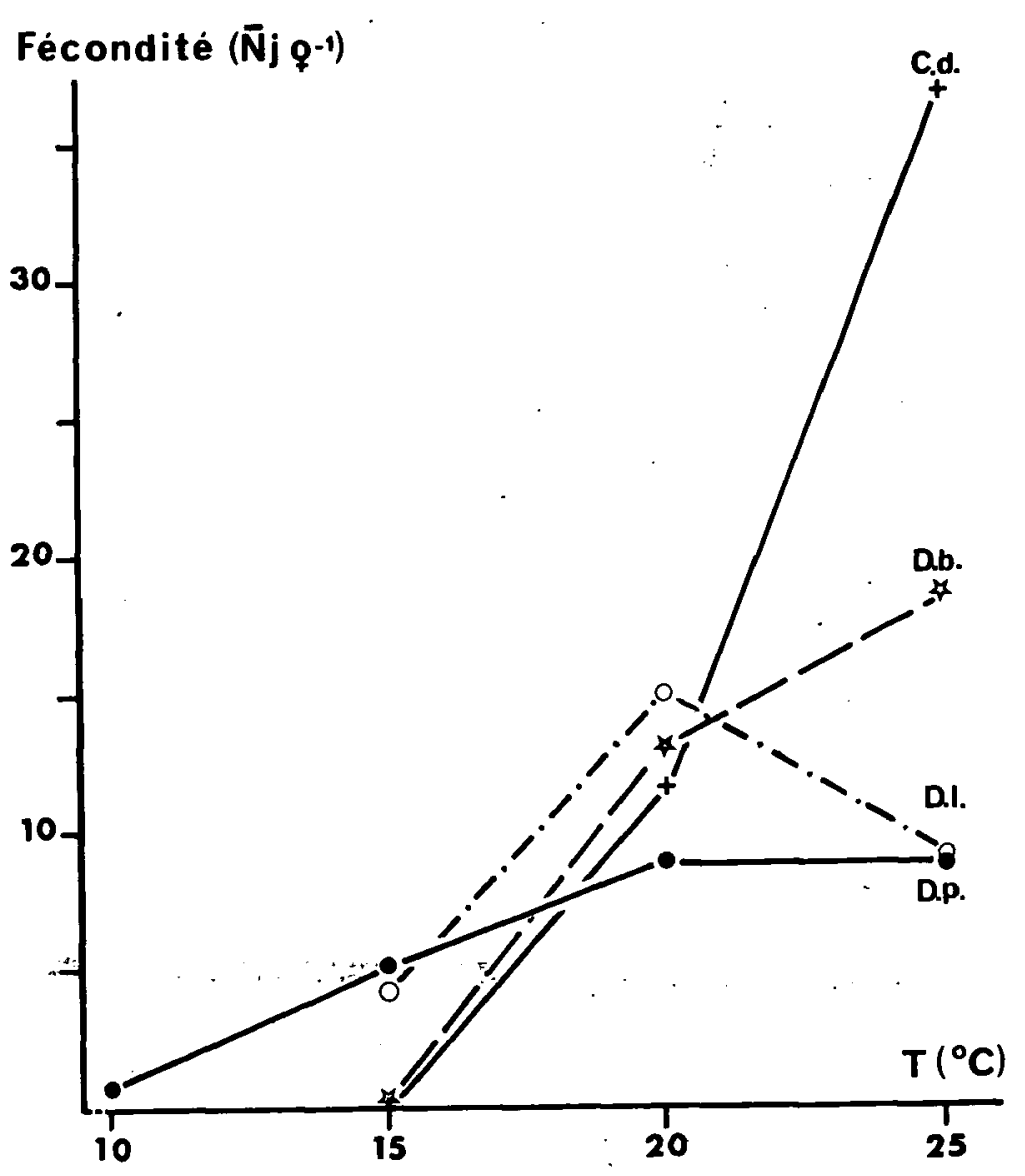

Fig. 6. Variation du nombre moyen de jeunes produits par une femelle durant sa vie en fonction de la température chez quatre espèces de Cladocères. Mêmes symbole que Fig .1.

Fig. 6. Variations of the mean number of of fsprings produced by a female during her life time in relation to temperature in four cladoceran species. Symbols as in Fig. 1.

observation ainsi que l'existence d'individus de taille $\geqslant 2 \mathrm{~mm}$ sont vraisemblablement liées à un faible taux de prédation par les poissons dont il n'existe pas d'espèces strictement planctonophages.

\section{Remerciements}

Les auteurs remercient G. Lacroix et $P$. Testard pour la relecture du manuscrit et pour leurs suggestions, O. Cherifi et A. Chifaa pour les données phytoplanctoniques.

\section{Travaux cités}

Borgmann U., Shear H. \& Moore J. 1984. - Zooplankton and potential fish production in Lake Ontario. Can. J. Fish. Aquat. Sci., 4 : 1303-1309.

Bottrell H.H., Duncan A., Gliwicz Z.B., Grygierek E., Herzig A., Hillbricht-Ilkowska A., Kurusawa H., Larsson P. \& Weglenska T. 1976. - A review of some problems in zooplankton production studies. Norw. J. Zool., 24 : 419-456.

Cherifi O. 1992. - Evolution et dynamique du phytoplancton en relation avec certains paramètres biotiques et abiotiques dans la retenue Lalla Takerkoust (Maroc). D.E.S., Faculté des Sciences Semlalia, Marrakech : 155 p. 
Cowgill U.M., Keating K.I. \& Takahashi I.T. 1985. - Fecundity and longevity of Ceriodaphnia dubia / affinis in relation to diet at two different temperatures. J. Crust. Biol., $5: 420-429$.

Gras R. \& Saint-Jean L. 1976. - Durée du développement embryonnaire chez quelques espèces de Cladocères et de Copépodes du lac Tchad. Cah. O.R.S.T.O.M., sér. Hydrobiol., $10: 233-254$.

Gras R. \& Saint-Jean L. 1978. - Durée et caractéristiques du développement juvénile de quelques Cladocères du lac Tchad. Cah. O.R.S.T.O.M., sér. Hydrobiol., 12 : 119-136.

Hanazato T. \& Yasuno M. 1985. - Effect of temperature in the laboratory studies on growth, egg development and first parturition of five species of Cladocera. Jap. J. Limnol. , 46 (3), 185-191.

Herzig A. 1984. - Temperature and life strategies of Diaphanosoma brachyurum, an experimental study on development, growth and survival. Arch. Hydrobiol., 101 : 143-178.

Lynch M. 1978. - Complex interactions between natural coexploiters Daphnia and Ceriodaphnia. Ecology, 59 : 552-564.

Lynch M. 1980. - The evolution of cladoceran life histories. Q. Rev. Biol., $55: 23-42$.

Lynch M. 1989. - The life history consequences of resource depression in Daphnia pulex. Ecology, $70: 246-256$.

Mc Cauley E., Murdoch W.W., Nisbet R.M. 1990. - Growth, reproduction, and mortality of Daphnia pulex Leydig : life at low food. Funct. Ecol., 4 : 505-514.
Mourelatos S. \& Lacroix G. 1990. - In situ filtering rates of Cladocera : effect of body length, temperature, and food concentration. Limnol. Oceanogr., 35 : 1101-1111.

Mourelatos S., Rougier C. \& Lacroix G. 1992. - Radiotracer losses due to freezing in formalin of ${ }^{14} \mathrm{C}$-labelled cladocerans. Arch. Hydrobiol., $126: 239-253$.

Pace M.L., Porter K. \& Feig Y.S. 1984. - Life history variation within a parthenogenetic population of Daphnia parvula (Crustacea : Cladocera). Oecologia, $63: 43-51$.

Peters R.H. 1983. - The ecological implications of body size. Cambridge Univ. Press : $329 \mathrm{p}$.

Reynolds C.S: 1984. - The écology of freshwater phytoplankton. Cambridge Univ. Press : $384 \mathrm{p}$.

Romanovsky Y.E. 1984. - Prolongation of postembryonic development in experimental and natural cladoceran populations. Int. Rev. ges. Hydrobiol., 69 : 149-157.

Schwartz S.S. 1984. - Life history strategies in Daphnia : a review and predictions. Oikos, $42: 114-122$.

Taylor B.E. 1985. - Effects of food limitation on growth and reproduction of Daphnia. Arch. Hydrobiol. Beih., Ergebn. Limnol., 21 : 285-296.

Threkeld S.T. 1987. - Life history strategies and resources allocation patterns. Mem. Ist. Ital. Idrobiol., 45 : 353-366. 